\title{
Aplicación del autoconcepto al desarrollo de la marca personal. Análisis comparativo entre estudiantes internacionales
}

\author{
Eva García Montero \\ Universidad Camilo José Cela \\ egarmo@ucjc.edu \\ Marián DE LA Morena TABOAdA \\ Universidad Camilo José Cela \\ mmorena@ucjc.edu \\ África Presol Herrero \\ Universidad Camilo José Cela \\ apresol@ucjc.edu
}

\begin{abstract}
Resumen:
El auge de las nuevas tecnologías ha posibilitado la creación de la marca personal a nivel individual. Cualquier persona haciendo uso de las redes sociales puede difundir su imagen o marca a fin de construir una imagen diferenciada. El interés por el desarrollo de una marca personal consolidada se ha incrementado en la medida que resulta más difícil hacerse valer en un mercado de trabajo cada vez más competitivo. Los estudiantes a punto de entrar en el mercado laboral son unos sujetos especialmente interesados en el desarrollo de su marca personal. Así esta investigación utiliza como muestra a estudiantes de dos nacionalidades: españoles y belgas. El objetivo es doble, por un lado, presentar el autoconcepto como una herramienta adecuada al desarrollo de la marca personal y por otro, establecer posibles diferencias entre el nivel de autoconcepto de ambas nacionalidades. En este artículo se van presentar los resultados obtenidos en un estudio sobre autoconcepto y marca personal llevada a cabo en Bélgica y en España entre enero y mayo de 2013.
\end{abstract}

Palabras Clave: Marca personal; autoconcepto; internacional; redes sociales.

\section{Self- concept and personal Brand development. Comparative analysis in international student.}

\begin{abstract}
:
Individual personal brands have experienced a growing importance due to the massive use of new technologies. Everyone can benefit from the use of social networks to make a personal brand spread establishing a difference with the rest of the individuals. The interest to develop a personal brand is growing partly because of the high competitive employment market. Students in their last university years are especially interested in developing a personal brand. This study shows results obtained in a sample of students from Belgium and Spain. The objectives are to analyze self-concept as a valid tool to develop a personal brand and to establish possible differences in the self-concept levels of both nationalities. This article shows the results obtained in a study about personal brand and self-concept carried out in Belgium and Spain in May 2013.
\end{abstract}

Key Words: Personal brand; self-concept; international; social networks. 


\section{Referencia normalizada:}

García Montero, E., De la Morena Taboada, M. y Presol Herrero, A. (2014): Aplicación del autoconcepto al desarrollo de la marca personal. Análisi comparativo entre estudiantes internacionales. Historia y Comunicación Social. Vol. 19. Núm. Especial Febrero. Págs. 819-833.

Sumario: 1. Introducción, 1.1. Autoconcepto como constructo, 1.2. Identidad personal y grupal, 1.3. Autoconcepto e identidad social, 1.4. Instrumentos de medición del autoconcepto, 2. Marca personal, 3. Método, 3.1 Muestra, 3.2 Instrumento, 3.3. Procedimiento, 4. Resultados, 5. Conclusiones, 6. Referencias bibliográficas, 7. Anexo

\section{Introducción}

\subsection{Autoconcepto como constructo}

El autoconcepto es entendido como una construcción de lo que el individuo percibe y valora de sí mismo y que se conforma en el marco de diversas experiencias socio-culturales a lo largo de todo el ciclo vital.

Resulta así ser la imagen mental que nos hacemos de nosotros mismos con sus defectos y virtudes. El autoconcepto es un constructo complejo que se genera no sólo a partir de la interacción con el YO, sino que depende también de la interacción con los demás. La compresión empírica de la idea del YO puede rastrearse a lo largo de toda la historia de la psicología. La experiencia del YO como objeto de atención es el estado psicológico que se conoce como autoconcepto. Este estado psicológico invita a la reflexión sobre las propias acciones para comprender hasta qué punto dichas acciones concuerdan con los valores y creencias personales así como con los del grupo (Carver y Scheier, 1981).

El autoconcepto como constructo comprende dimensiones cognitivas, afectivas, y conductuales (Alonso García y Román Sánchez, 2003). El mismo posee a su vez relevancia para comprender la interacción del individuo con su ambiente, dado que cumple funciones referidas a la autorregulación de la conducta, la automotivación, y la autoeficacia, etc (Gónzalez Pienda, Núñez Pérez, Gzlez Pumariega y García García, 1997) cuestiones vinculadas con el desarrollo de la imagen personal que queremos proyectar a nuestro entorno.

Mientras que el autoconcepto se define como una conciencia unitaria y continua de quienes somos (Baumeister, 1998), queda menos claro cómo debemos entender la identidad social, puesto que puede ser tan variada como lo sean los grupos a los que pertenecemos. Cada uno de nosotros posee un número de identidades sociales diferentes que se superponen y que incluyen aquellas derivadas de la pertenencia a grupos claramente delimitados, como por ejemplo, profesor de comunicación y otras más amplias e incluso ambiguas como, por ejemplo, ciudadano europeo. Una 
consecuencia importante de esto son los diferentes autoconceptos que pueden emerger dependiendo de cuál de estas identidades sobresalga (Crisp y Hewstone, 2001). En este estudio se ha analizado también la variable país como elemento diferenciador en la valoración del autoconcepto y de la identidad social. Podemos decir que en la medida en que se vea afectada por las características y procesos del grupo variará la identidad social de cada miembro del grupo. La investigación ha demostrado que las personas perciben su grupo y otros grupos sociales en términos diferentes dependiendo del grupo con el que se comparan o del dominio en el que se establece la comparación (Van Rijswijk y Ellemers, 2002).

Por lo tanto no deberíamos concebir determinadas identidades sociales como inherentemente positivas o negativas sino que variará en función del grupo o de la dimensión con la que se establezca la comparación. Por ejemplo, un estudiante de Arquitectura puede establecer una identidad positiva en términos de inteligencia si se compara con estudiantes de Bellas Artes y negativa si se compara con estudiantes de Ingeniería. Si se compara en términos de creatividad podría establecer lo contrario, negativa con los primeros y positiva con los segundos.

\subsection{Identidad personal y grupal}

Darwin dejó claro que la supervivencia de las especies dependía de su capacidad de adaptación al entorno. Esta cuestión se complica algo más en la especie humana. Seres sociales por naturaleza la capacidad de supervivencia de la especie humana está conectada con nuestra capacidad de adaptarnos al medio social. Esta adaptación social ha tenido como consecuencia la aparición de comportamientos que nos habiliten como buenos miembros del grupo (Baumeister y Leary, 1995) que están conectados con la auto-regulación y el autoconcepto.

Tener conciencia del nosotros mismos nos sirve para mantener las habilidades sociales básicas que garanticen relaciones positivas con otros miembros del grupo. Para ser aceptado socialmente es preciso tener una conciencia de los propios pensamientos, emociones y comportamientos que nos permita alterarlos o modificarlos para satisfacer los estándares o expectativas del grupo. Según Heatherton (2010) podemos hablar de al menos cuatro componentes psicológicos necesarios para una buena integración en el grupo: autoconcepto, mentalización (capacidad de inferir los estados mentales de los demás para predecir acciones futuras (Amodio y Frith, 2006, Gallagher y Frith, 2003, Mitchell, 2006) lo que nos permite empatizar con los demás y predecir sus opiniones), detección de amenazas (nos permite predecir posibles riesgos de exclusión del grupo. Si los seres humanos tenemos una necesidad básica de ser aceptados por el grupo debe existir un mecanismo que nos permita detectar el status de pertenencia (Macdonald y Leary, 2005) y auto-regulación (nos permite corregir los comportamientos que provocan exclusión así como actuar proactivamente para evitar su aparición ya que también se auto regula para promover la consecución de logros (Higgins, 1997)). 
En nuestra investigación nos centraremos en el estudio del autoconcepto desde la doble perspectiva de la aceptación del grupo y de la diferenciación positiva encaminada a la construcción de una marca personal.

\subsection{Autoconcepto e identidad social}

Como decíamos es el contexto social más que las características concretas del grupo el que determina el matiz de la evaluación positiva o negativa. Así, en este estudio tratamos de extraer conclusiones acerca de la identidad que establecemos en función de la valoración del grupo. Para la creación de una marca personal tenemos que tener en cuenta estos elementos no fijos que varían en función de la dimensión y del grupo con el que se establece la construcción de identidad. Más que tratar de decidir si el autoconcepto individual es más importante que el social creemos que es interesante especificar las condiciones bajo las cuales uno tiene más probabilidades de sobresalir sobre el otro y con cuáles consecuencias. La creación de una marca personal tiene que ver con el contexto social en la medida en que necesitamos ser reconocidos y diferenciados positivamente por el grupo. Según Sánchez Burón una de las características de los seres humanos y de los grupos formados por ellos es la necesidad de comparación entre las personas o grupos para valorar sus niveles de ejecución (Sánchez Burón, 1998). Esta comparación social busca la obtención de resultados positivos con la finalidad de afirmar la imagen de uno mismo dentro del propio grupo. Este sentido de pertenencia genera en las personas una identidad social que se deriva de las características del grupo de pertenencia y tiene como consecuencia que las personas integradas en un grupo realicen continuamente comparaciones, ya sea con miembros de su mismo grupo o miembros de grupos cercanos para comprobar el nivel de ejecución sobre las características representativas de su grupo (Sánchez Burón, 1998). Este estudio propone esta comparación del autoconcepto con el concepto que tiene el grupo de la identidad personal del sujeto evaluado ya que el autoconocimiento integrado en el sistema cognitivo hace referencia a la comparación con otras personas para conocerse adecuadamente. Normalmente este objetivo personal conduce a la comparación entre personas similares.

Relacionado con este objetivo está el de auto-verificación que tiene que ver con el deseo de mantener y consolidar la idea que se tiene de sí mismo, mostrando una consistencia del comportamiento en las distintas situaciones en las que se pueda presentar. Esta auto-verificación conecta con la necesidad de coherencia a la hora de definir y construir nuestra marca personal.

\subsection{Instrumentos de medición del autoconcepto}

En la actualidad, disponemos de diversas escalas para evaluar el autoconcepto (Clerici y García, 2010). Las más utilizadas son las de Fitts (1965), Harter (1985), Piers (1984), Marsh y Hattie (1996) y Musitu, Garcia y Gutierrez (1994). Todas ellas asumen el constructor como multidimensional. 
La "Piers-Harris Children's Self-concept Scale" en particular evalúa cinco dimensiones del autoconcepto infantil mediante cinco subescalas: Conducta, Estatus intelectual y escolar, Apariencia y atributos físicos, Ansiedad, Popularidad, y Felicidad y satisfacción. La subescala Estatus intelectual y escolar refleja la percepción del niño acerca de su comportamiento social. La subescala Estatus intelectual y escolar refleja la autoapreciación acerca de su rendimiento en las tareas escolares. La subescala Apariencia y atributos físicos contemplan las apreciaciones del niño hacia su propio cuerpo. La subescala Ansiedad brinda una perspectiva acerca de sus estados de humor, incluyendo una variedad de emociones específicas. La subescala Popularidad arroja información acerca de la percepción de sus habilidades sociales y del reconocimiento que los otros hacen de su propia conducta. Finalmente la subescala Felicidad y satisfacción refleja sentimientos de satisfacción o insatisfacción personal. Los puntajes en estas cinco subescalas, sumados a otros ítems no agrupados brindan un puntaje total que permite definir la valoración del niño hacia sí mismo de modo global.

\section{Marca personal}

Hay pocos términos tan de moda en la actualidad. Término heredado del marketing no supone más que la aplicación de todo lo que hemos aprendido acerca de la gestión de las marcas a la gestión de nosotros mismos considerándonos un producto que debe ser elegido en un mercado competitivo.

Para entender el concepto "marca personal" hay que empezar por la palabra "marca" que procede el término anglosajón "Brand", que ha derivado en "Branding" o lo que es lo mismo el proceso de construir y administrar la marca.

Tom Peters, el autor norteamericano al que se le atribuye la autoría del concepto formal de branding personal o marca personal, utiliza el término en su artículo The Brand Called You (Peters, 1997). En él afirma que la única manera de diferenciarnos en un mercado tan competitivo, es tratar de identificarnos e incluso vender nuestras cualidades como las empresas lo hacen con sus productos.

Así pues, la marca personal es el resultado de desarrollar un proceso que tiene por objetivo la identificación de aquello que resulta valioso, fiable y útil con la propia persona. Es decir, hay que descubrir ¿Quiénes somos? ¿Qué sabemos hacer? ¿Que ofrecemos? ¿Qué necesidades satisfacemos? ¿Qué defendemos? ¿Cómo mostramos todo eso al mundo exterior?

La marca personal rompe con el paradigma clásico de adaptarse a las oportunidades que se nos presentan, se trata de descubrir qué oportunidades son compatibles con nosotros y atraerlas, sin tener que perseguirlas. 
Asimismo, la marca personal amplía el concepto de la persona a largo plazo, es decir todas las acciones que vayamos ejecutando se irán acumulando, teniendo consecuencias en nuestro futuro.

Aunque existen diferencias significativas entre el concepto de marca comercial y personal, nos centramos en el concepto de marca personal, valor empleado en la investigación, que en muchos casos se convierte en marca comercial.

Para responder al por qué de la importancia de crear marca personal, haciendo referencia al creador del concepto formal, Tom Peters, decir que es necesario diferenciarse del resto de personas. La necesidad de destacar en el mercado laboral en el que lo que importa es la imagen de cada uno hace necesario diferenciar y potenciar nuestras cualidades al igual que lo hacen los productos de las marcas.

Para ello, es necesario saber construir la propia marca personal y que ser capaces de hacerla perdurar en el tiempo. Además la marca personal incrementa su importancia en un mundo en el que las relaciones interpersonales son cada vez más anónimas y se nos conoce tan sólo por nuestro currículo vitae a través de medios digitales.

La intención del "Personal Branding" para individuos o grupos, se resume en ser recordados, tenidos en cuenta, resultar la opción preferente y por supuesto, ser elegidos entre la competencia.

Al igual que en la creación de imagen corporativa, en términos generales las etapas de creación de marca personal se dividen en (Collell López, Recolons Argenter, Micó, Hortal Rosell, Brun Sánchez y Herms Vázquez, 2011):

- Autoconocimiento. Análisis de las creencias, valores o principios, habilidades y competencias. Se analizan las motivaciones, el diagnóstico de la situación actual, los miedos, zonas de confort.

- En esta etapa nos preguntamos ¿Quién nos conoce?, ¿Somos capaces de transmitir lo que somos?, ¿Nos gusta cómo nos ven?, ¿Sabemos lo que queremos?, ¿Nos gusta la vida que llevamos?, ¿Estamos dispuestos a cambiar?

- Estrategia. En esta etapa hay que definir la visión, misión y valores, un mensaje central, unos objetivos, públicos objetivos y un plan de acción diferente para lograr los objetivos a alcanzar. Se responden a preguntas como ¿Dónde queremos llegar al final de nuestra vida?, ¿Cómo queremos ser en ese momento?, ¿Tenemos las habilidades necesarias?, ¿Qué vale la pena cambiar y mantener?, ¿Qué mensaje queremos transmitir?, ¿A quién queremos llegar?, ¿Cuáles son los tiempos, cuáles son los atajos?

- Comunicación. Hay que decidir los medios adecuados para hacer que el mensaje llegue, con mayor alcance y con soluciones más tácticas, tanto en entornos reales como virtuales.

En esta comunicación sugerimos que la marca personal es algo mucho más complejo que utilizar un blog para darse a conocer, o una red social para ponerte localizar contactos, se trata de un proceso estratégico denominado "branding perso- 
nal "o "personal branding" en el que se gestiona la propia marca y que parte de la fase de autoconocimiento, sigue con fase en la que se trabaja la estrategia y culmina con una fase de visibilidad y comunicación en la que sí tienen una cabida especial las nuevas tecnologías.

Por ello la marca personal será creada, difundida y protegida con la intención de diferenciarse, alcanzando mayor éxito en los medios en los que uno decida hacerse visible.

\section{Método}

\subsection{Muestra}

El cuestionario se aplicó en Bélgica a alumnos de comunicación del Plantijn Hogeschool en Amberes y del Erasmus University College en Bruselas. En ambos casos se aplicó a tres sujetos de una clase que fueron evaluados por treinta compañeros de aula. En España se aplicó en la Facultad de Comunicación de la UCJC a tres alumnos que también fueron evaluados, cada uno, por treinta compañeros de clase.

\subsection{Instrumento}

Para nuestra investigación hemos creado un cuestionario de autoconcepto con cinco dimensiones (CIFES). Hemos utilizado como base el cuestionario de Piers-Harris para alumnos de 7 a 12 años haciendo las correcciones oportunas para adaptarlo a nuestra muestra (alumnos universitarios de entre 18-25 años) y a los objetivos de nuestra investigación, esto es, que la medición del autoconcepto sea una herramienta para determinar el autoconocimiento que requiere la implementación de la marca personal.

En nuestro cuestionario hemos utilizado cinco dimensiones basadas en las dimensiones de Piers-Harris con las siguientes correcciones:

- Autoconcepto conductual: No sólo la percepción de portarse de forma apropiada en diferentes tipos de situaciones (Me porto bien en clase) también implica la capacidad de hacer cosas bien (Soy mañoso).

- Autoconcepto intelectual: Percepción de competencia en situaciones no sólo escolares si no en situaciones en las que se aprenden cosas nuevas, que requieren un desafío intelectual (Soy listo, Tengo buena memoria....)

- Autoconcepto físico: Percepción de apariencia (Tengo buen tipo, Me gustan mis ojos) y competencia física (Raramente estoy enfermo).

- Autoconcepto emocional: Percepción de ausencia de problemas de tipo emocional, más bien de poseer una adecuado equilibrio emocional (Me enfado poco, Soy una persona equilibrada). 
- Autoconcepto social: Percepción del éxito en las relaciones con los otros (Se puede confiar en mi, Caigo bien a los demás).

La dimensión felicidad-satisfacción no se ha utilizado por no considerarla necesaria para determinar la marca personal.

El cuestionario consta de 30 frases, seis por cada dimensión, en formato de diferencial semántico frente a las 80 del cuestionario Piers-Harris con respuesta dicotómica si/no. Consideramos más adecuado el formato diferencial semántico pues nos permitía matizar más la imagen que los demás tienen del sujeto que normalmente no es un todo o nada sino una escala de grises.

\subsection{Procedimiento}

Se elaboraron dos formatos de cuestionario. Por un lado el propiamente de autoconcepto, donde el sujeto se autoevaluaba y reportaba lo que él creía que era en realidad, no lo que creía ser ni lo que le gustaría ser. Por otro lado, el cuestionario para el grupo, el que rellenaban los demás para evaluar los mismos ítems que evaluaba el sujeto. De esta manera obteníamos resultados acerca de lo que el sujeto opinaba sobre él mismo (autoconcepto) y lo que opinaban los demás.

Los cuestionarios no cuentan con instrucciones porque en todos los casos se aplican personalmente por parte del investigador que procede a dar las indicaciones oportunas a los sujetos.

El cuestionario de autoconcepto se aplica a un sujeto y a 30 compañeros de aula que reportan acerca de lo que piensan de él en cada uno de los ítems, esto es, reportan acerca de la imagen que tienen de él.

Debido al número de sujetos a quienes hemos aplicado el cuestionario, nuestro estudio se encuentra en una fase explorativa. En esta fase pretendemos dos objetivos: por un lado pre-testar el cuestionario y por otro detectar si hay tendencias que merezca la pena confirmar con investigaciones sucesivas.

En una fase posterior se ampliará la muestra de sujetos a los que se aplica el cuestionario de autoconcepto así como la muestra de sujetos conocidos del mismo. Se pasará no sólo a compañeros de aula, sino, también, a familiares y conocidos en otros ámbitos, para conseguir una imagen del sujeto más amplia.

\section{Resultados}

Analizando los datos extraídos podemos ver como hay una clara diferencia entre el autoconcepto de los alumnos belgas y los españoles: 
Comparativa autoconcepto e imagen

Gráfico 1. Alumnos belgas

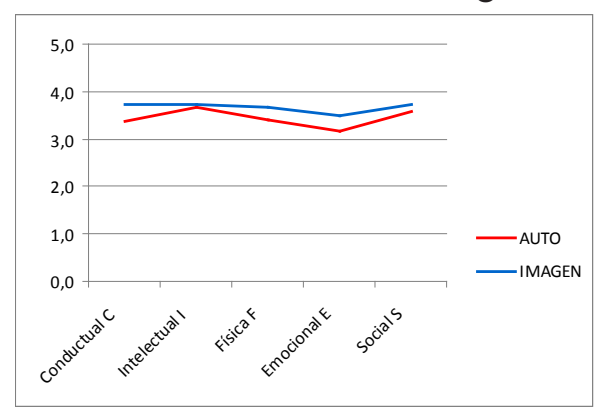

Gráfico 2. Alumnos españoles

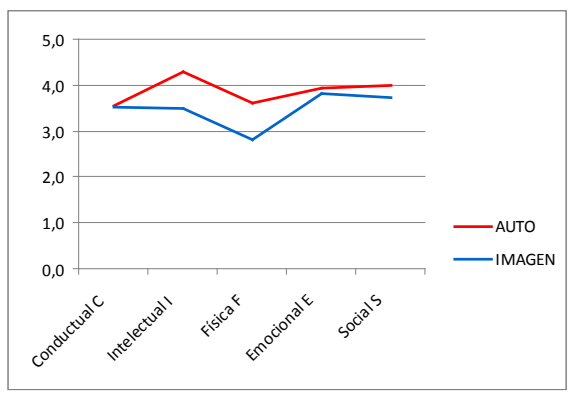

Los alumnos belgas tienen ligeramente un autoconcepto más pobre de ellos mismos que los españoles los cuales se puntúan a ellos mismos de manera más positiva de lo que lo hacen sus compañeros de aula.

Gráfico 3. Comparativa autoconcepto e imagen Alumnos belgas

\begin{tabular}{|c|c|c|} 
& AUTO & IMAGEN \\
\hline Conductual C & 3,4 & 3,7 \\
Intelectual I & 3,7 & 3,7 \\
Física F & 3,4 & 3,7 \\
Emocional E & 3,2 & 3,5 \\
Social S & 3,6 & 3,7 \\
\hline
\end{tabular}


Gráfico 4. Comparativa autoconcepto e imagen Alumnos españoles

\begin{tabular}{|c|c|c|} 
& AUTO & IMAGEN \\
\hline Conductual C & 3,6 & 3,5 \\
Intelectual I & 4,3 & 3,5 \\
Física F & 3,6 & 2,8 \\
Emocional E & 3,9 & 3,8 \\
Social S & 4,0 & 3,7 \\
\hline
\end{tabular}

Si analizamos más en detalle las gráficas por dimensiones vemos como en el caso de los alumnos belgas la diferencia entre autoconcepto e imagen no es muy elevada. Prácticamente no hay diferencia en las percepciones personales y la de los demás en la dimensión intelectual.

Comparativa autoconcepto e imagen en la dimensión intelectual

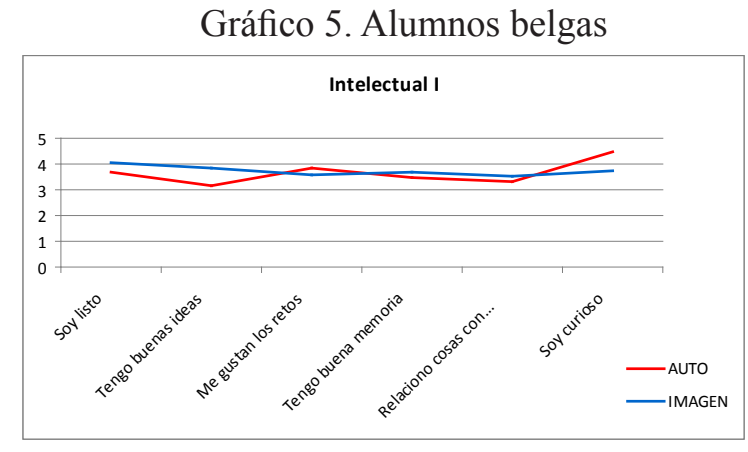

Gráfico 6. Alumnos españoles

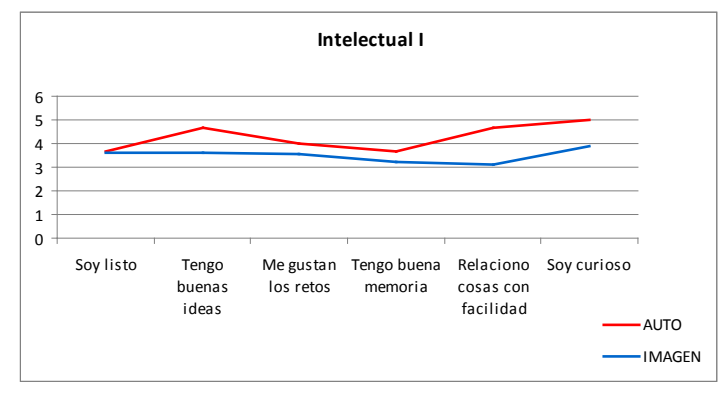

En cambio, en el caso de los alumnos españoles las diferencias más significativas están en las dimensiones física e intelectual, siendo la emocional y la conductual las dimensiones en las que están más parejas lo que el sujeto cree de sí mismo y lo que creen los demás. 
Gráfico 7. Comparativa autoconcepto e imagen en la dimensión física. Alumnos españoles

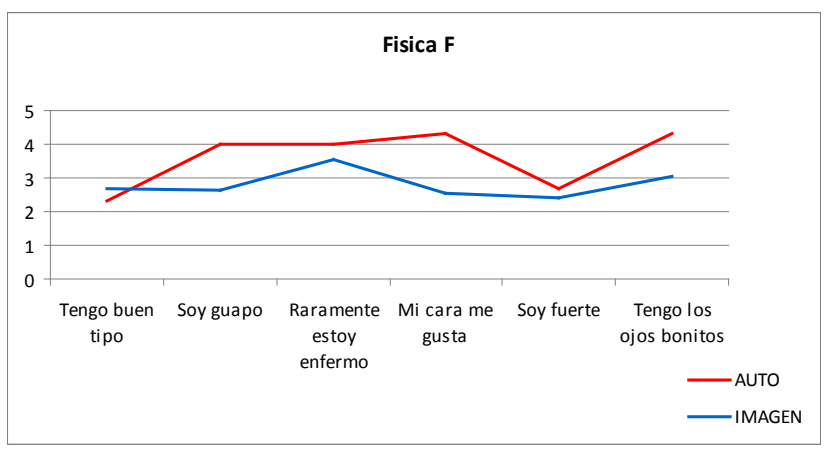

Gráfico 8. Comparativa autoconcepto e imagen en la dimensión emocional. Alumnos españoles

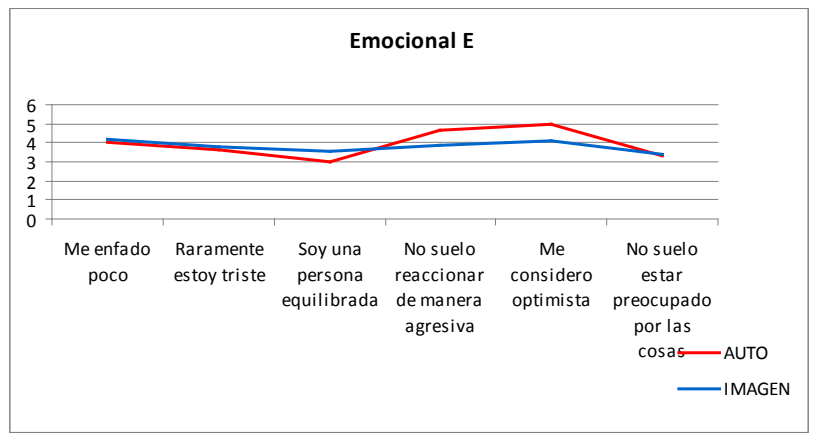

Gráfico 9. Comparativa auto-concepto e imagen en la dimensión social. Alumnos españoles

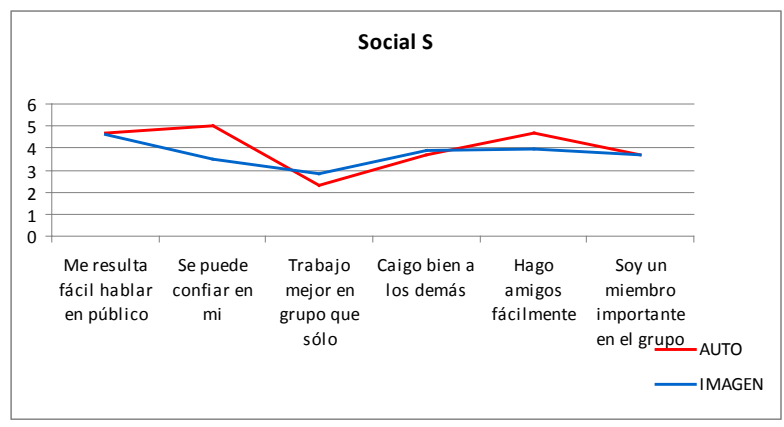


Aunque estos datos deben refrendarse por una muestra más amplia lo cierto es que los primeros resultados apuntan a que la nacionalidad podría determinar la manera de entender el auto-concepto. Los belgas parecen más modestos que los españoles.

Por otro lado, si consideramos el autoconocimiento como una premisa inicial para el planteamiento de la marca personal la manera de entender la creencia de uno mismo determinaría el enfoque de nuestra marca personal.

De cualquier manera es preciso ampliar la investigación a un número mayor de sujetos para confirmar estos extremos.

\section{Conclusiones}

Para construir una marca personal es necesario partir de un autoconocimiento que posibilite detectar aquellos aspectos que nos permiten diferenciarnos y aportar valor. Si entendemos por marca personal aquello que nos hace únicos y diferentes del resto encontramos una conexión entre el autoconcepto y la marca personal. El poder de la marca está relacionado con la capacidad de influir no de ejercer la fuerza. Las marcas personales más poderosas son aquellas que quedan en los caminos poco transitados, por eso descubrir y desarrollar una marca personal requiere tiempo, esfuerzo, paciencia y método (Pérez Ortega, 2012).

En la actualidad, se cuenta con diversas escalas para evaluar el autoconcepto. Todas ellas asumen al constructo como multidimensional puesto que además del reconocimiento de nuestros propios estados mentales la integración armoniosa en grupos sociales requiere la habilidad de interpretar los estados mentales y emocionales de los demás (Heatherton y Krend1, 2009).

Parece que en una sociedad tan competitiva como la actual puede ser aconsejable que el sujeto se trate a sí mismo como lo haría con un producto. Esto es, que trabaje su imagen a fin de destacar entre los demás y pueda alcanzar sus objetivos. Este transferir lo que hemos aprendido en marketing al individuo ha dado como resultado el llamado personal branding.

El proceso del personal branding parte del autoconocimiento del sujeto al que se aplica después una estrategia de mejora y de visibilidad que da como resultado un nuevo posicionamiento en la mente de los demás.

Una de las herramientas de las que dispone el sujeto para realizar el briefing de sí mismo, para cumplimentar la fase de autoconocimiento, son los test de autoconcepto. En esta comunicación proponemos un nuevo test de auto-concepto adaptado a la finalidad del personal branding. Este test de autoconcepto se completa con el test de imagen que rellenan los miembros del grupo en el que está inserto el sujeto y que nos permite establecer la diferencia entre lo que es y cómo los demás le perciben a fin 
de modificar ambas dimensiones para adaptarlas a la consecución de los objetivos de posicionamiento, la adscripción positiva al grupo y la consecución de logros y metas.

La variable autoconcepto muestra una dimensión fluida en la medida que puede cambiar en función de los diferentes roles que el sujeto asume en su vida diaria. Parece entonces recomendable hacer un análisis de la imagen que tienen los demás no de una manera agregada sino por sectores. En este estudio analizamos la imagen que tenían del sujeto el resto de compañeros de aula, considerando exclusivamente el rol de estudiante del sujeto. En futuras investigaciones sería interesante incluir la imagen que el resto de grupos tienen de nuestro sujeto en sus distintos roles: hijo, amigo, trabajador, miembro de un club, etc.

\section{Bibliografía}

6.1 Capítulos o artículos en libros o revistas en papel:

ALONSO GARCÍA, J. y ROMAN SANCHEZ, J. (2003). Educación familiar y autoconcepto en niños pequeños. Madrid: Editorial Pirámide.

AMODIO, D.M., FIRTH, C.D. (2006) Meeting of the minds: the medial frontal cortex and social cognition. Nature 7, 268-77.

BAUMEISTER ,R.F. (1998). The Self. In The Handbook of Social Psychology, ed. D.T. Gilbert, S.T. Fiske, G. Lindzey, 680-740. Boston MA: McGraw-Hill.

BAUMEISTER, R.F, Leary M.R. (1995). The need to belong: desire for interpersonal attachments as a fundamental human motivation. Psychological Bulletin, $117,497-529$.

CARVER C, SCHEIER M. (1981). Attention and Self-Regulation. New York: Springer-Verlag.

CASULLO, M.M. (1990). El autoconcepto. Técnicas de evaluación. Buenos Aires: Psicoteca Editorial.

CRISP, R.J., HEWSTONE, M. (2001). Multiple categorization and implicit intergroup bias: differential category dominance and the positive-negative asymmetry effect. European Journal of Social Psychology 31, 45-62.

ESNAOLA, I.; GOÑI, A. Y MADARIAGA, J.M. (2008). El autoconcepto: perspectivas de investigación. Revista de Psicodidáctica, vol.13, N ${ }^{\circ} 1$ (pp.179-194).

FITTS, W.H. (1965). The Tennessee self-Concept Scale. Nashville, TN.: Counselor Recordings and Test.

GONZALEZ PIENDA,, J.A.; NUÑEZ, J.C.; GLEZ PUMARIEGA, S.; GARCIA GARCIA, M.S. (1997). Autoconcepto, autoestima y aprendizaje escolar. Psicothema, vol.9, n'2 (pp. 271-289)

GALLAGHER, H.L, FIRTH, C.D. (2003). Functional imaging of "theory of mind". Trends in Cognitive Scences, 7, 77-83.

HARTER, S. (1985). Manual for the self-perception profile for children. (Revision of the perceived competence scale for children). Denver, CO. University of Denver. 
HEATHERTON, T.F. (2010). Building a social brain. In Social Neuroscience: Toward Understanding the Underpinnings of the Social Mind, ed. A. Todorov, S.T. Fiske, D. Prentice. New York: Oxford University Press.

HEATHERTON, T.F., RENDELL, A.C. (2009). Social emotions: neuroimaging. In Encyclopaedia of Neuroscience, ed. L Squire, 9, 35-39. London: Academic Press.

HIGGINS, E.T. (1997). Beyond pleasure and pain. American Pschycologist, 52, 128-300.

MACDONALD, G., LEARY, M.R. (2005). Why does social exclusion hurt? : The relationship between social and physical pain. Pschycological Bulletin, 131,20223.

MARSH, H.W. Y HATTIE, J. (1996). Theoretical perspectives on the structure of self-concept. En B. A. Bracken (Ed), Handbook of self-concept. (pp. 38-90). Nueva York: Wiley.

MITCHELL, J.P. (2006). Mentalizing and Marr: An information processing approach to the study of social cognition. Brain Research, 1079, 66-75.

MUSITU, G.; GARCÍA, F. Y GUTIÉRREZ, M. (1994). Autoconcepto Forma A. Manual. Madrid: TEA.

PÉREZ ORTEGA, A. (2012). Marca Personal: Cómo convertirse en la opción preferente. Madrid: ESIC editorial.

PETERS, T. (1997). La marca que llama. Fast Company, 10 (10)

PIERS, E. (1984). The Piers-Harris Children's self-concept scale (The way I feel about myself) revised manual. Los Ángeles, CA: W. P. S.

SÁNCHEZ BURÓN, A. (1998). El sexo como variable estímulo: análisis de las interacciones entre los sexos. En Género y Sociedad, ed. J Fernández. Madrid: Ediciones Pirámide.

VAN RIJSWIJK, W., ELLEMERS, N. (2002). Context effects on the application of stereotype content to multiple categorizable targets. Personality and Social Psychology Bulletin, 28, 90-101.

6.2 Artículos en publicaciones web:

COLLELl LÓPEZ, J., RECOLONS ARGENTER G., MICÓ, P.A., HORTAL ROSELL, P., BRUN SÁNCHEZ, I. Y HERMS VÁZQUEZ, A. (2011) Personal y Transferible. Un año de marca personal intensiva. Recopilación de artículos del blog www.soymimarca.com entre octubre 2010 y octubre 2011. Soymimarca, S.L. http:// www.soymimarca.com/acerca-de/nuestro-libro/

CLERICI, G. Y GARCÍA, M.J. (2010). Autoconcepto y percepción de pautas de crianza en niños escolares. Aproximaciones teóricas. Anuario de investigaciones, versión online vol.17. (pp. 205-212). http://www.scielo.org.ar/scielo.php?scrip$\mathrm{t}=$ sci arttext\&pid=S1851-16862010000100065

SCHAWBEL, D. (2009). Marca personal 101: Cómo descubrir y crear su marca. News Social Media y Web Consejos. 2009. Web. O6deseptiembre 2010.http:// mashable. com2009/02/05/personal-branding-101. 


\section{Las autoras}

Eva García Montero es doctora en Ciencias Sociales por la Universidad Camilo José Cela, licenciada en Psicología Industrial por la UNED y licenciada en Publicidad y Relaciones Públicas por la Universidad Complutense. También cuenta con un título de Especialista en Gestión Publicitaria por esta última Universidad.

Ha desarrollado su carrera profesional en agencias de publicidad (McCann-Erickson y J. Walter Thompson) y departamentos de marketing de empresas, lo que compagina con su labor como docente en la Universidad Camilo José Cela de Madrid donde imparte asignaturas de Relaciones Públicas y Psicología de la Comunicación. Es profesora invitada en Plantjin I Hogeschool Amberes (Bélgica), Artevelde Hogeschool Gante (Bélgica) y Erasmus Hogeschool Bélgica.

Sus líneas de investigación y conferencias están vinculadas con la inteligencia emocional, las técnicas de comunicación oral y la aplicación de las TIC a la docencia.

Marián de la Morena Taboada es profesora de la Universidad Camilo José Cela desde el año 2000. Licenciada en Filología inglesa por la UCM y Máster en Dirección de Marketing y Gestión Comercial por ESIC es doctora acreditada en Ciencias Sociales y de la Educación. Ejerce como docente en las Facultades de Ciencias de la Comunicación y Ciencias Sociales y de la Educación. Es profesora invitada de las universidades Plantijn, Artevelde y Erasmus Hogeschool de Bélgica donde ha impartido seminarios sobre marca personal y redes sociales. Su experiencia profesional se ha desarrollado en ámbitos internacionales que incluyen la embajada de los EE.UU. en Madrid y el Departamento de Atención al cliente del Deutsche Bank en Wiesbaden, Alemania. Ha participado en numerosos congresos nacionales e internacionales donde ha presentado comunicaciones sobre redes sociales, marca personal, motivación y estrategias de aprendizaje.

África Presol Herrero es doctora en Publicidad y Relaciones Públicas por la Universidad Camilo José Cela. Licenciada en Ciencias de la Información en la rama de Publicidad y Relaciones Públicas por la Universidad Complutense de Madrid (UCM). CAP por el Instituto de Ciencias de la Educación en la UCM.

Especialista en Diseño Gráfico actualmente es docente en la Facultad de Comunicación de la Universidad Camilo José Cela donde imparte las asignaturas de Creatividad, Documentación, Comunicación e imagen corporativas, Teoría y práctica de la Publicidad y Diseño Gráfico Publicitario. En su bagaje profesional cuenta con haber trabajado para cuentas como Buena Vista International-Disney, Publirama, Talante de Comunicación y Oracle. Sus líneas de investigación giran en torno al análisis del mensaje publicitario, al desarrollo de técnicas de estimulación de la creatividad y a la publicidad On line en concreto a las nuevas formas de comunicación en los medios. 